\title{
Multiuser Diversity and Multiplexing using Multiple Random Beams in Wireless Systems
}

\author{
Sung-Soo Hwang and Yong-Hwan Lee
}

\begin{abstract}
In this paper, we propose a new multiple-antenna transmission scheme that can simultaneously achieve both diversity and multiplexing gain in the multi-user domain, by using multiple random beams. Multiple beams are generated so that the users encounter multiple channels at the same time, enabling the use of multi-user diversity through each channel. Although the signal-to-noise power ratio (SNR) of each channel is reduced in proportion to the number of beams, multiple beams are generated so that the multiplexing gain is larger than the decrease of SNR, increasing the overall system capacity.
\end{abstract} sity.

Index Terms-Multiple beams, multiplexing, multiuser diver-

\section{INTRODUCTION}

$\mathbf{T}$ HE next generation transmission system should be able to provide high data rate multimedia services to users in mobile, nomadic and fixed environment. The nature of multimedia services may need the downlink capacity much larger than the uplink. In recent years, the capacity of wireless systems has been increased significantly with the development of two advanced technologies; the use of multiple antennas known as MIMO [1]-[4] and packet scheduling known as opportunistic scheduling or multi-user diversity (MUD) [6][14].

The capacity of MIMO systems can be increased linearly in proportion to the number of antennas in rich scattering channel environment. The MIMO system can improve the capacity by increasing the diversity gain or spatial multiplexing gain using multi-antenna channels. Space-time coding (STC) is a typical MIMO diversity scheme [3]. Diagonal Bell laboratories layered space-time (D-BLAST), vertical Bell laboratories layered space-time (V-BLAST) scheme and MIMO with singular value decomposition (SVD) are typical MIMO multiplexing schemes [1], [2], [4]. However, there is a traditional trade-off issue between the diversity and multiplexing gain [5]. It may not be easy to get both full diversity and multiplexing gain simultaneously. In addition, the diversity and multiplexing gain can substantially be reduced depending on the channel condition.

The MUD is a novel scheme that exploits independent channel characteristics of each user [6]-[14], [20]. It allows a

Manuscript received August 25, 2004; revised June 19, 2005 and October 19, 2005; accepted October 24, 2005. The associate editor coordinating the review of this paper and approving it for publication was Y.-C. Liang.

S.-S. Hwang is with the Telecommunication R\&D Center, Samsung Electronics, Dong Suwon P. O. Box 105, Suwon-city, Gyeonggi-do, 442-600, Korea (e-mail: mygarlic@gmail.com).

Y.-H. Lee is with the School of Electrical Engineering and Computer Science, Seoul National University, Kwanak P.O. Box 34, Seoul 151-600, Korea (e-mail: ylee@snu.ac.kr).

Digital Object Identifier 10.1109/TWC.2007.04587. user in the best channel condition to send the signal, achieving a system capacity larger than that in additive white Gaussian noise (AWGN) channel with the same signal-to-noise power ratio (SNR) [9]-[11]. However, when the channel gain has a small fluctuation and/or varies slowly as in fixed wireless or nomadic channels, the MUD may not provide significant improvement of the capacity. To overcome this problem, the base station can utilize multiple antennas with randomly generated weights, known as opportunistic or random beamforming [11], [12]. It was reported that the opportunistic beamforming can provide a diversity gain larger than the STC [9], [11]. Moreover, it can provide a diversity gain even when the channel is completely correlated because the channels of each user are still independent. However, the opportunistic beamforming does not fully utilize the multiplexing and diversity in the multi-antenna domain, but it only exploits the multi-user diversity.

Recently, simultaneous use of multi-user diversity and multi-antenna (spatial) multiplexing has been considered [12]. It is a combination of MIMO SVD and multi-user diversity using random beamforming. However, it is applicable only to MIMO systems, not to multi-input single output (MISO) systems. Moreover, its multiplexing gain is limited by the rank of the channel matrix of each user. As a result, it cannot provide a multiplexing gain when the rank of channel matrix is one (e.g., in completely correlated channel). Besides, it involves a very large implementation complexity since all the receivers should carry out the SVD process and send back many parameters to the base station.

In this paper, we propose a new multi-antenna transmission scheme that can simultaneously provide multi-user diversity and multiplexing (MUDAM) gain, using multiple random beams. If we can have multiple channels simultaneously by generating multiple beams, we can get both the diversity and multiplexing gain in the multi-user domain. Since the transmitted power is spilt into multiple channels, the SNR of each channel is reduced inversely proportional to the number of multiple beams. However, total system capacity can be increased by increasing the multiplexing gain much more than the decrease of the SNR. The multiplexing scheme using multiple transmit antennas can be considered as a combination of transmission beamforming and "dirty paper" pre-coding method [15]-[17]. However, these previous schemes require perfect channel state information (CSI) of all users. The use of multiple "orthogonal" beams was briefly discussed in [11] and this scheme was improved with partial CSI in [20]. However, its capacity gain can be less than the use of a single beam when the number of users is small. Besides it needs 
multiple pilot signals in proportion to the number of beams and its capacity increases very slowly as the number of users increases. We consider the generation of multiple beams in a random manner under the constraint that multiple beams interfere with each other at a controlled level, although they may not be orthogonal. The proposed scheme can improve the capacity even in completely correlated channels since it utilizes independent channels between different users. The proposed scheme is applicable to both the MISO and MIMO systems.

This paper is organized as follows. In Section II, we introduce the system model including the multi-user diversity scheme and random beamforming. A MUDAM scheme using multiple random beams is proposed in Section III. It is also described how to generate such multiple random beams. The performance of the proposed MUDAM scheme is verified by computer simulation in Section IV. Finally, Section V concludes this paper.

\section{SySTEM MODEL}

Consider an $(M \times 1)$ MISO system, where the base station has $M$ transmit antennas and each of $K$ users has 1 receive antenna. The received signal of the $k$-th user at time $t$ can be represented as

$$
y_{k}(t)=\mathbf{h}_{k}^{H}(t) \mathbf{w}(t) s(t)+z_{k}(t), \quad k=1,2, \ldots K
$$

where $\mathbf{h}_{k}^{H}(t)=\left[h_{1, k}^{*}(t), h_{2, k}^{*}(t), \ldots h_{M, k}^{*}(t)\right]$ denotes the impulse response of the MISO channel and $h_{m, k}^{*}(t)$ represents the channel from the $m$-th transmit antenna to the $k$-th user. $\mathbf{w}(t)=\left[w_{1}(t), w_{2}(t), \ldots w_{M}(t)\right]^{T}$ is the weight vector of the beamformer and $s(t)$ is the user signal. $z_{k}(t)$ is a complex circular-symmetric Gaussian processes with zero mean and the variance $\sigma_{z}^{2}$. Here, the superscript $*$ and $H$ denote complex conjugate and conjugate of the transpose, respectively. The channel $h_{m, k}^{*}(t)$ is flat fading and is assumed to remain constant within a block. The channels of each user are independent and the transmit power is fixed to $P$ at all times, i.e., $E\left\{\|\mathbf{w}(t) s(t)\|^{2}\right\}=P$, where $E\{x\}$ denotes the expectation of $x$. Assume that $P=1$ in this paper. We also assume that instantaneous channel quality information such as the SNR is available at the base station. The base station assigns the channel resource to a user in the best channel quality at each time, exploiting the user diversity.

The weight $\mathbf{w}(t)$ can be generated in a random manner as

$$
w_{m}(t)=\sqrt{\alpha_{m}(t)} e^{j \theta_{m}(t)}, \quad m=1,2, \ldots, M
$$

where $\alpha_{m}(t)$ and $\theta_{m}(t)$ are random processes in the range of $0 \leq \alpha_{m}(t) \leq 1$ and $0 \leq \theta_{m}(t) \leq 2 \pi$. We assume that $\sum_{m=1}^{M}\left|\sqrt{\alpha_{m}(t)}\right|^{2}=1$ for normalization of the total transmit power.

Since the effective channel response of the $k$-th user is given by

$$
\begin{aligned}
h_{k}^{*}(t) & =\mathbf{h}_{k}^{H}(t) \mathbf{w}(t) \\
& =\sum_{m=1}^{M} h_{m, k}^{*}(t) \sqrt{\alpha_{m}(t)} e^{j \theta_{m}(t)},
\end{aligned}
$$

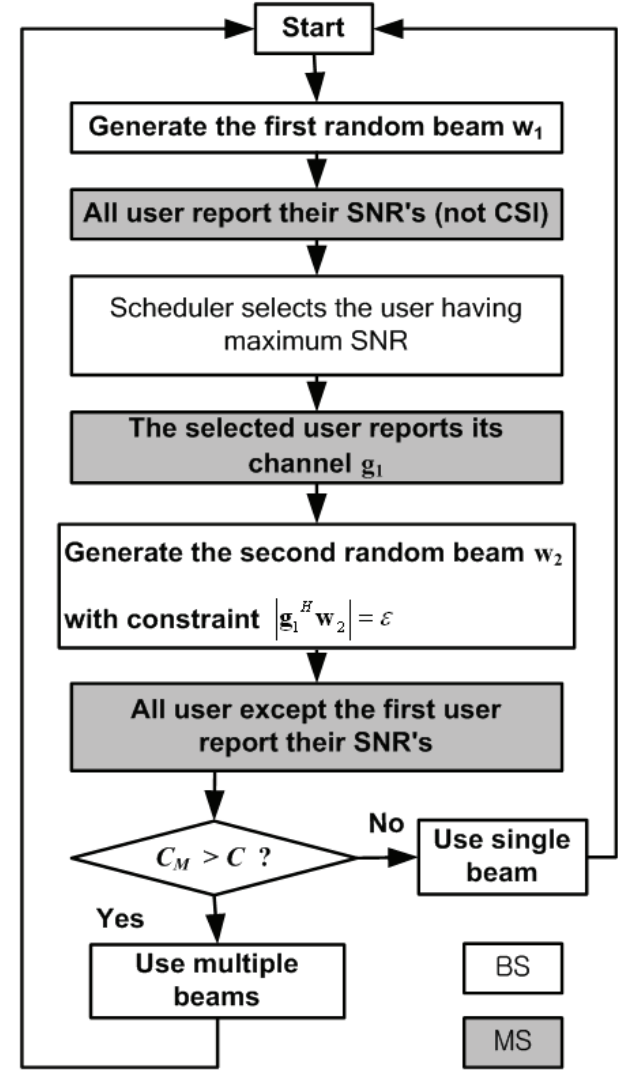

Fig. 1. Example of the procedure of the proposed MUDAM scheme.

it can be controlled by adjusting $\alpha_{m}(t)$ and $\theta_{m}(t)$. The MUD gain increases by inducing a larger fluctuation in the effective channel. Although the capacity of the STC in the MISO system is bounded by that in AWGN channel, the random beamforming scheme can outperform the SISO system in AWGN channel by exploiting the MUD [9], [10]. However, the channel fluctuation may not be increased by a random beamforming method when the channel has Rayleigh fading.

\section{Multi-User Diversity And Multiplexing (MUDAM)}

We consider a MUD system with a novel multiplexing scheme in the multi-user domain, using multiple random beams. The term "random" is used since multiple beams are generated using a method similar to the random beamforming. Since the proposed scheme can achieve the multiplexing gain in the multi-user domain, not in the multi-antenna domain, it can also provide the multi-user multiplexing (MUM) gain regardless of the channel correlation.

The proposed scheme makes multiple beams in a successive manner, and the scheme only controls the interference from the latter beam to the former beam. For example, the procedure of the proposed MUDAM scheme in the case of two beams is shown in Fig. 1 and assume a $(2 \times 1)$ MISO system with two beams as illustrated in Fig. 2, where two signals $d_{1}(t)$ and $d_{2}(t)$ are multiplexed by weight $\mathbf{w}_{1}=\left[w_{1,1}, w_{2,1}\right]^{T}$ and $\mathbf{w}_{2}=\left[w_{1,2}, w_{2,2}\right]^{T}$, yielding a transmitted signal

$$
\mathbf{x}(t)=\mathbf{w}_{1}(t) d_{1}(t)+\mathbf{w}_{2}(t) d_{2}(t) .
$$




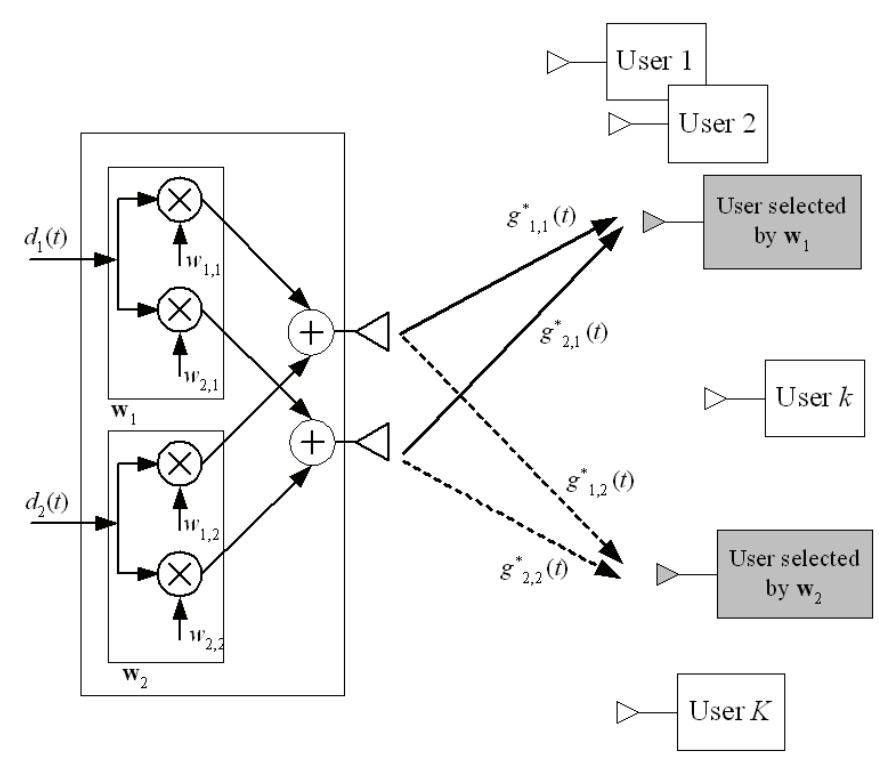

Fig. 2. The proposed MUDAM scheme applied to a $(2 \times 1)$ MISO system.

First, the base station generates a random beam weight $\mathbf{w}_{1}$ (as in the opportunistic beamforming) and each user reports the SNR to the base station. The SNR of user $k$ is given by $\left|\mathbf{h}_{k}^{H} \mathbf{w}_{1}\right|^{2} / \sigma_{k}^{2}$ where $\sigma_{k}^{2}$ is the noise power of user $k$. The base station selects a user having the maximum SNR

$$
\gamma=\max _{k \in\{1,2, \ldots K\}}\left\{\frac{\left|\mathbf{h}_{k}^{H} \mathbf{w}_{1}\right|^{2}}{\sigma_{k}^{2}}\right\}
$$

where $\gamma$ is the maximum SNR achieved through the first beam without the use of multi-beam. Assume that the base station selects user $p$ in this process. The selected user $p$ reports its channel response $\mathbf{h}_{p}(t)$ to the base station. Let $\mathbf{g}_{1}(t)=\mathbf{h}_{p}(t)$, where $\mathbf{g}_{1}(t)$ denotes the channel response corresponding to the first beam $\mathbf{w}_{1}$.

The base station can generates the second beam $\mathbf{w}_{2}$ so that it generates the interference to the user of $\mathbf{w}_{1}$ in a controlled manner, i.e.,

$$
\mathbf{g}_{1}^{H} \mathbf{w}_{2}=\varepsilon
$$

where $\varepsilon$ should be chosen to be small enough for the SINR of the first beam is nearly unaffected as

$$
\gamma_{1}=\frac{\frac{1}{2}\left|\mathbf{g}_{1}^{H} \mathbf{w}_{1}\right|^{2}}{\sigma_{p}^{2}+\frac{1}{2}|\varepsilon|^{2}}
$$

Note that the second beam $\mathbf{w}_{2}=\left[w_{1,2}, w_{2,2}\right]^{T}$ has two elements and Equation (6) has only one condition. Therefore, Equation (6) is an under-determined equation and $\mathbf{w}_{2}$ has many solutions. We can generate the second beam with a random variable $w_{1,2}$ and the solution of Equation (6). Therefore the second beam is also partially random beam.

After the base station selects the best user through the second beam, resulting SINR can be represented as

$$
\gamma_{2}=\max _{p \notin, k \in\{1,2, \ldots K\}}\left\{\frac{\frac{1}{2}\left|\mathbf{h}_{k}^{H} \mathbf{w}_{2}\right|^{2}}{\sigma_{k}^{2}+\frac{1}{2}\left|\mathbf{h}_{k}^{H} \mathbf{w}_{1}\right|^{2}}\right\} .
$$

Note that the transmit power of each beam is reduced inversely proportional to the number of multiple beams since the total

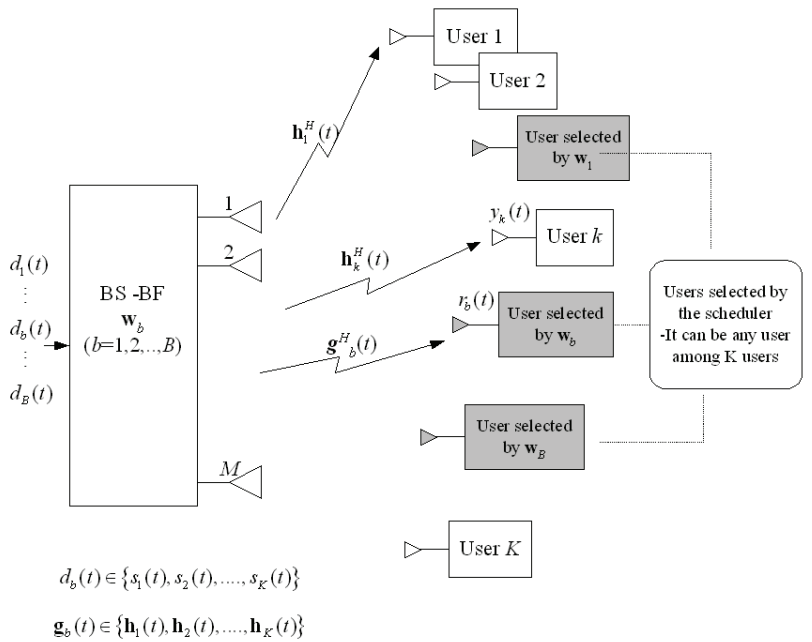

Fig. 3. A multi-user multiplexing system with multiple beams.

transmit power should be constant. Note that the interference from the second beam to the first beam user is controlled by $(1 / 2)|\varepsilon|^{2}$, but the interference $(1 / 2)\left|\mathbf{h}_{k}^{H} \mathbf{w}_{1}\right|^{2}$ from the first beam to the second beam user is not controllable. Thus, it is required for the base station to choose a user having the maximum SINR in an opportunistic manner.

Then the base station calculates the following capacities and decides whether it employs multi-beam or not.

$$
\begin{gathered}
C=\log _{2}(1+\gamma) . \\
C_{M=2}=\log _{2}\left(1+\gamma_{1}\right)+\log _{2}\left(1+\gamma_{2}\right) .
\end{gathered}
$$

If $C_{M}>C$ then the base station employs multi-beam and otherwise, employs only single beam.

Consider a generalized MUDAM structure as depicted in Fig. 3. The base station transmits $B$ signals $\left\{d_{1}(t), d_{2}(t), \ldots, d_{B}(t)\right\}$ to $K$ users through $B$ beams with weight vector $\left\{\mathbf{w}_{1}(t), \mathbf{w}_{2}(t), \ldots \mathbf{w}_{B}(t)\right\}$ at the same time. In this case, (1) can be rewritten as

$$
y_{k}(t)=\mathbf{h}_{k}^{H}(t) \sum_{b=1}^{B} \mathbf{w}_{b}(t) d_{b}(t)+z_{k}(t), \quad k=1,2, \ldots, K
$$

where $d_{b}(t)$ is the user signal such that

$$
d_{b}(t) \in\left\{s_{1}(t), s_{2}(t), \ldots, s_{K}(t)\right\}, \quad b=1,2, \ldots, B .
$$

We assume that each signal has the same power $\sigma_{s}^{2}$. Let $\mathbf{g}_{i}(t)$ denote the channel response of the best user through the $i$-th beam $\mathbf{w}_{i}(t)$, i.e.,

$$
g_{i}(t) \in\left\{\mathbf{h}_{1}(t), \mathbf{h}_{2}(t), \ldots, \mathbf{h}_{K}(t)\right\}, \quad i=1,2, \ldots, B
$$

Assume that the scheduler selects user $p$ (i.e., $k=p$ ) for the $i$-th beam (i.e., $b=i$ ). That is, the base station transmits the user signal $s_{p}(t)$ using the $i$-th beam w $i(t)$ (or $d_{i}(t)=s_{p}(t)$ and $\left.\mathbf{g}_{i}(t)=\mathbf{h}_{p}(t)\right)$. The received signal $r_{i}(t)$ through the $i$ th beam (i.e., the received signal of user $p$ ) can be represented 
as

$$
\begin{aligned}
r_{i}(t) & =y_{p}(t) \\
& =\mathbf{h}_{p}^{H}(t)\left[\mathbf{w}_{i}(t) s_{p}(t)+\sum_{b=1, \neq i}^{B} \mathbf{w}_{b}(t) d_{b}(t)\right] \\
+z_{p}(t) & =\mathbf{g}_{i}^{H}(t) \mathbf{w}_{i}(t) d_{i}(t) \\
& +\sum_{b=1, \neq i}^{B} \mathbf{g}_{i}^{H}(t) \mathbf{w}_{b}(t) d_{b}(t)+z_{p}(t)
\end{aligned}
$$

where the first term is the desired signal, the second term is the interference due to multi-beam multiplexing and the third term is additive noise.

The base station performs the successive controlling the power of interference as

$$
\mathbf{g}_{i}^{H} \mathbf{w}_{b}=\left\{\begin{array}{cc}
\varepsilon, & i<b \\
\mathbf{g}_{i}^{H} \mathbf{w}_{i}, & i=b \\
\times, & i>b
\end{array}\right.
$$

where $x$ denotes the amount of uncontrollable interference that varies depending on the situation and when $i=b$, no control is needed. The proposed MUDAM scheme generates the weight matrix $\mathbf{W}\left(=\left[\mathbf{w}_{1}, \mathbf{w}_{2}, \ldots, \mathbf{w}_{B}\right]\right)$ satisfying

$$
\mathbf{G}^{H} \mathbf{W}=\mathbf{F}
$$

where $\mathbf{G}$ is the channel matrix of the selected users defined by

$$
\mathbf{G}=\left[\mathbf{g}_{1}, \mathbf{g}_{2}, \ldots, \mathbf{g}_{B}\right]
$$

and $\mathbf{F}$ is a constraint matrix defined by

$$
\mathbf{F}^{\triangleq}\left[\begin{array}{ccccc}
\mathbf{g}_{1}^{H} \mathbf{w}_{1} & \varepsilon & \varepsilon & \ldots & \varepsilon \\
\times & \mathbf{g}_{2}^{H} \mathbf{w}_{2} & \varepsilon & & \varepsilon \\
\times & \times & \ddots & & \vdots \\
\vdots & & & \mathbf{g}_{B-1}^{H} \mathbf{w}_{B-1} & \varepsilon \\
\times & \times & \ldots & \times & \mathbf{g}_{B}^{H} \mathbf{w}_{B}
\end{array}\right] .
$$

Note that the time index $t$ is omitted for ease of description because the weight vector $\mathbf{w}_{i}(t)$ and the channel $\mathbf{g}_{i}(t)$ are assumed to unchanged during each slot time.

Fig. 4 illustrates the SINR of 39 users obtained by the proposed MUDAM scheme with two beams. It can be seen that the $21^{\text {st }}$ and $6^{\text {th }}$ user have the best SINR through the first and second beam, respectively. Thus, the transmitter sends the $21^{\text {st }}$ and $6^{\text {th }}$ user signal using the first and second beam, respectively (i.e., $\mathbf{g}_{1}(t)=\mathbf{h}_{21}(t)$ and $\mathbf{g}_{2}(t)=\mathbf{h}_{6}(t)$ ).

Now let's consider the generation of such multiple beams in a random manner. We assume that the channel condition is unchanged during the feedback process as in the opportunistic beamforming.

The base station generates the first beam weight $\mathbf{w}_{1}=$ $\left[\mathbf{w}_{1,1}, \mathbf{w}_{2,1}, \ldots, \mathbf{w}_{M, 1}\right]$ in a random manner as

$$
w_{m, 1}=\sqrt{\alpha_{m, 1}} e^{j \theta_{m, 1}}, \quad m=1,2, \ldots, M
$$

where $\alpha_{m, b}$ and $\theta_{m, b}, b=1, \ldots, B$, are time-variant random processes over a time slot, having a value between 0 and 1 , and 0 and $2 \pi$, respectively. Let $g_{1}$ be the impulse response of the channel of the first user selected by the scheduler.

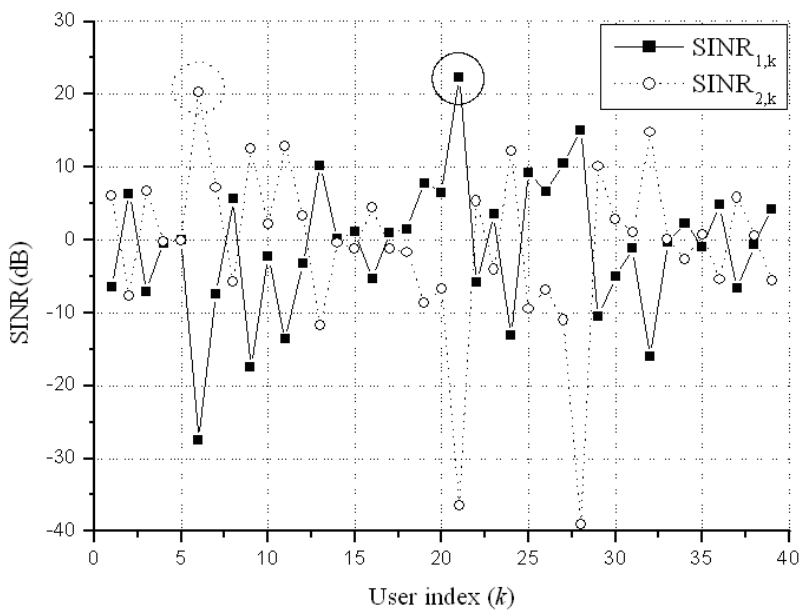

Fig. 4. The SINR of each user in a $(2 \times 1)$ MISO system with two random beams.

Then, the base station generates the next random beam weight $\mathbf{w}_{2}$ such that $\mathbf{g}_{1}^{H} \mathbf{w}_{2}=\varepsilon$. Here, we assume that $\varepsilon_{i}=\varepsilon$ for all $i$. Note that the scheduler does not need the channel information of all the users, but only that of the selected users. Since the weight $\mathbf{w}_{2}$ is an $M$-dim vector with a single constraint (i.e., an under-determined system), the rest $(M-1)$ elements of $\mathbf{w}_{2}$ can be determined by (19). Thus, we need to solve a single equation satisfying

$$
\mathbf{g}_{1}^{H} \mathbf{w}_{2}=\varepsilon
$$

or

$$
\sum_{m=1}^{M-1} g_{m, 1}^{*} \sqrt{\alpha_{m, 2}} e^{j \theta_{m, 2}}+g_{M, 1}^{*} w_{M, 2}=\varepsilon
$$

Then, we have Equation (22), where the constant $1 / \sqrt{p_{b}}$ is for normalization, making $\left\|\mathbf{w}_{b}\right\|^{2}=1$.

Similarly, the weight of the $b$-th beam can be generated by determining $(M-b+1)$ elements randomly and the rest $(b-1)$ elements are determined by solving equations,

$$
\mathbf{g}_{i}^{H} \mathbf{w}_{b}=\varepsilon, \quad i=1,2, \ldots, b-1
$$

where

$$
\mathbf{w}_{b}=\left[\begin{array}{c}
\mathbf{w}_{\text {rand }} \\
\mathbf{w}_{\text {sol }}
\end{array}\right]
$$

$\left(\mathbf{w}_{\text {rand }}\right)_{m}=\frac{1}{\sqrt{p_{b}}} \cdot \sqrt{\alpha_{m, b}} e^{j \theta_{m, b}}, \quad m=1,2, \ldots, M-b+1$

and also Equation (26). In [11], [20], multiple "orthogonal" beams are simultaneously generated such that

$$
\mathbf{W}^{H} \mathbf{W}=\mathbf{I}
$$

where $\mathbf{I}$ is an identity matrix. Note that the "orthogonal" beam scheme does not consider the separation of effective channels $\mathbf{g}_{b}^{H} \mathbf{w}_{b}$, but only the separation of beams $\mathbf{w}_{b}, b=1, \ldots, B$. As a result, unlike the proposed MUDAM scheme, the orthogonal multiple beam scheme can provide a MUM gain only when the users are separated by orthogonal beams. Besides, it requires the use of multiple pilot signals equal to the number of orthogonal beams. 


$$
\mathbf{w}_{m, 2}=\left\{\begin{array}{cc}
\frac{1}{\sqrt{p_{2}}} \cdot \sqrt{\alpha_{m, 2}} e^{j \theta_{m, 2}}, & m=1,2 \ldots, M-1 \\
\frac{1}{\sqrt{p_{2}}} \cdot \frac{1}{g_{M, 1}^{*}} \cdot\left[\varepsilon-\left(\sum_{m=1}^{M-1} g_{m, 1}^{*} \sqrt{\alpha_{m, 2}} e^{j \theta_{m, 2}}\right)\right], & m=M
\end{array}\right.
$$

$$
\mathbf{w}_{\text {sol }}=\frac{1}{\sqrt{p_{b}}}\left[\begin{array}{ccccc}
g_{M-b+2,1}^{*} & g_{M-b+3,1}^{*} & \cdots & g_{M-1,1}^{*} & g_{M, 1}^{*} \\
g_{M-b+2,2}^{*} & g_{M-b+3,2}^{*} & \cdots & g_{M-1,2}^{*} & g_{M, 2}^{*} \\
\vdots & & & & \vdots \\
g_{M-b+2, b-2}^{*} & & & \ddots & g_{M, b-2}^{*} \\
g_{M-b+2, b-1}^{*} & g_{M-b+3, b-1}^{*} & \cdots & g_{M-1, b-1}^{*} & g_{M, b-1}^{*}
\end{array}\right]\left[\begin{array}{c}
\varepsilon-\sum_{m=1}^{M-b+1} g_{m, 1}^{*} \sqrt{\alpha_{m, b}} e^{j \theta_{m, b}} \\
\varepsilon-\sum_{m=1}^{M-b+1} g_{m, 2}^{*} \sqrt{\alpha_{m, b}} e^{j \theta_{m, b}} \\
\vdots \\
\varepsilon-\sum_{m=1}^{M-b+1} g_{m, b-1}^{*} \sqrt{\alpha_{m, b}} e^{j \theta_{m, b}}
\end{array}\right]
$$

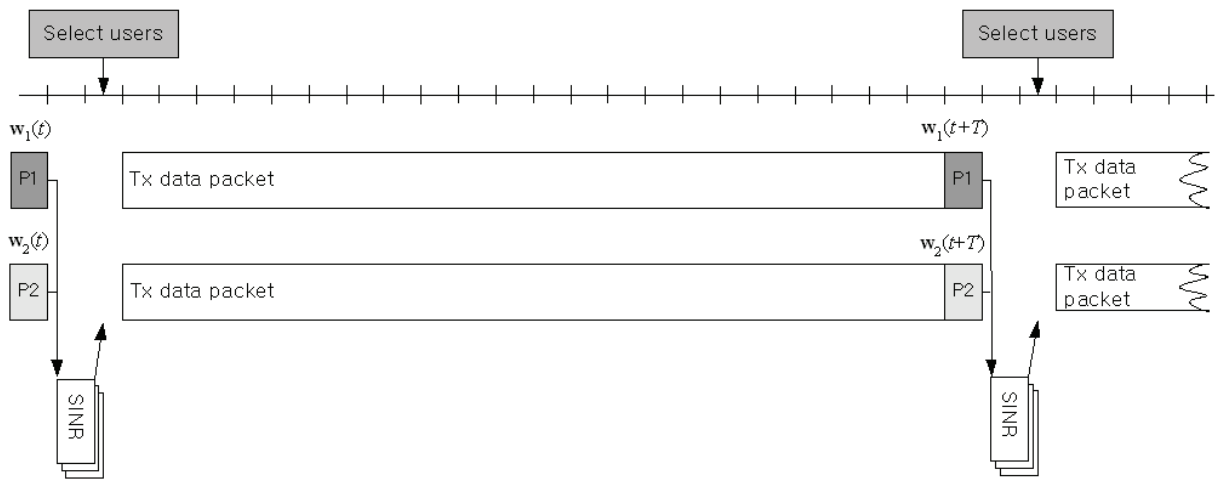

(a) The orthogonal beam scheme.

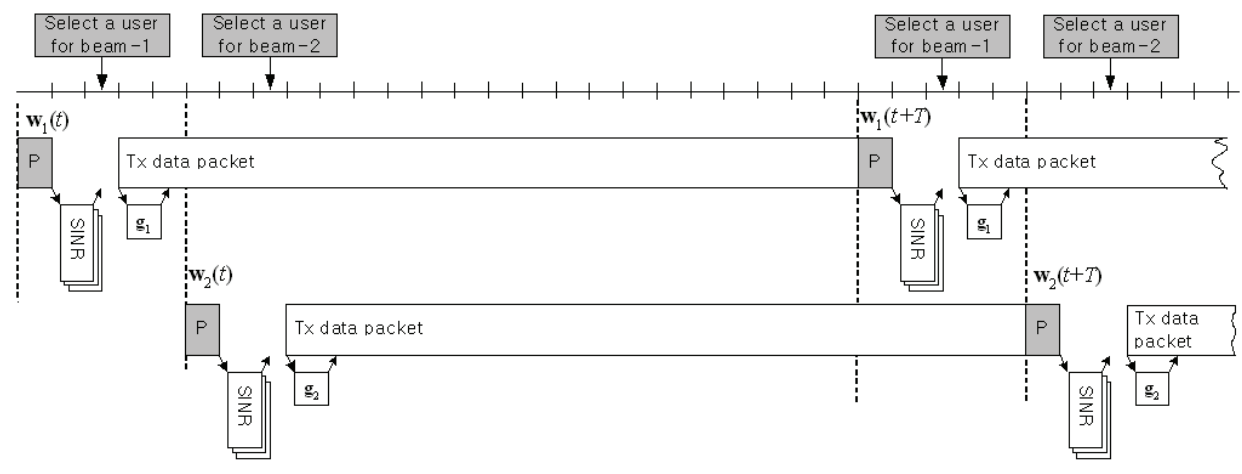

(b) The proposed MUDAM scheme.

Fig. 5. Generation of multiple beams in a $(2 \times 1)$ MISO system.

Fig. 5 illustrates the generation of multiple beams in the two $(2 \times 1)$ MISO schemes. The orthogonal beam scheme needs multiple pilot signals (e.g., P1 and P2 in Fig. 5) for simultaneous generation of orthogonal beams. Thus, the mobile station needs an additional processor to detect these multiple pilot signals. On the other hand, the proposed MUDAM scheme can generate multiple beams in a successive manner. Thus, the same pilot signal can be used for all beams. Note that the proposed MUDAM scheme needs only one channel response $\mathrm{g}_{b}$ from the selected user, not all the users.

Fig. 6 depicts the capacity of $(2 \times 1)$ MISO systems in Rayleigh fading channel at $20 \mathrm{~dB}$ SNR where $\varepsilon=0.01$ for the MUDAM scheme. The analytic capacity is obtained using the results of the Appendix. It can be seen that the use of the proposed MUDAM scheme always outperforms the single beam scheme.

\section{Performance Evaluation}

The performance of the proposed MUDAM scheme is verified by computer simulation. The simulation results are obtained by averaging over 1000 independent channel realizations per user. We assume that channels of all the users are mutually independent and have the same average SNR. It is assumed that the channel has flat Rayleigh fading.

Fig. 7 compares the performance of the proposed MUDAM, opportunistic beamforming scheme [11] (denoted as "single beam") and multiple orthogonal beam scheme [20] (denoted as "orthogonal"), when a $(2 \times 1)$ MISO system is employed in Rayleigh fading channel at $20 \mathrm{~dB}$ SNR. The proposed MUDAM scheme is designed with $\varepsilon=0.01$. It can be seen that the proposed MUDAM scheme always provides a capacity larger than the single beam and orthogonal beam schemes. It 


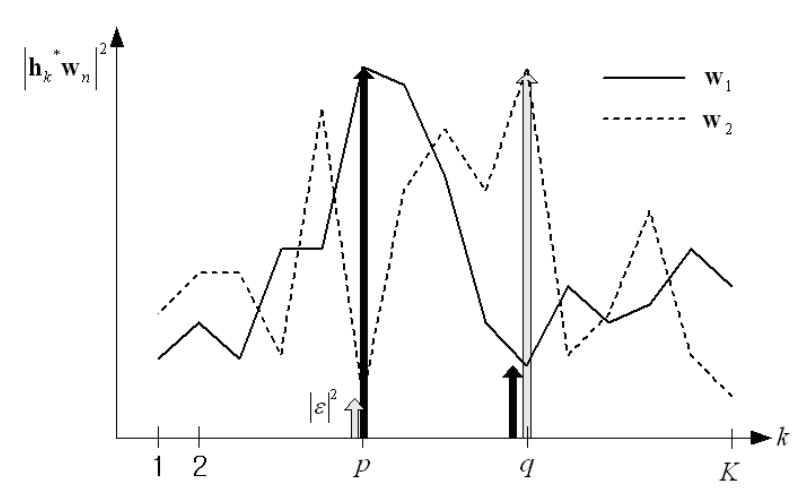

(a) The proposed MUDAM scheme.

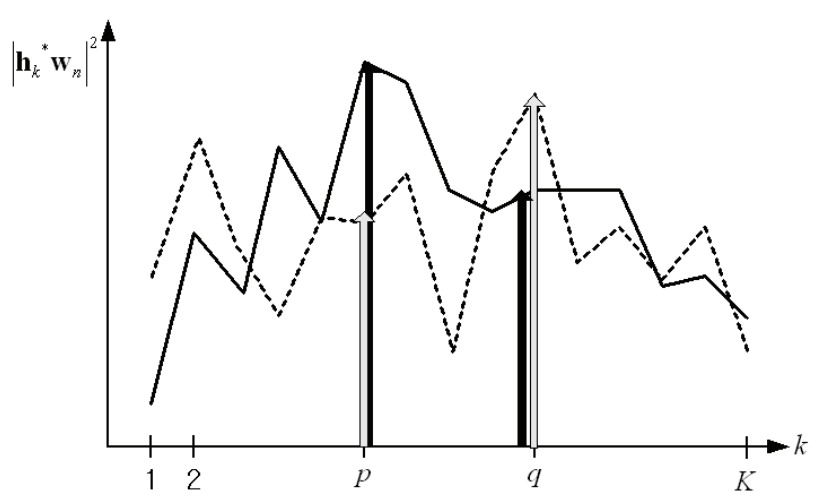

(b) The orthogonal scheme.

Fig. 8. The gain profile of each beam in $(2 \times 1)$ MISO systems.

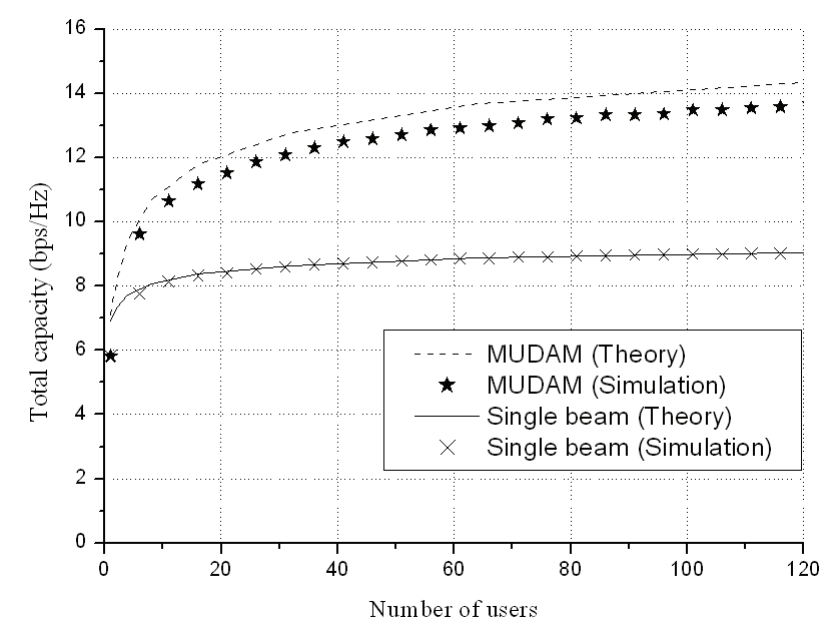

Fig. 6. The analytic and simulation result of $(2 \times 1)$ MISO systems in Rayleigh fading channel at 20dB SNR.

can also be seen that the orthogonal scheme cannot provide a MUM gain when the number of users is small. This is mainly due to the fact that the orthogonal scheme cannot guarantee the separation of multiple users. As an example, Fig. 8 illustrates the gain of each beam in a $(2 \times 1)$ MISO system with two beams as a function of each user index. Fig. 8(a) illustrates that the proposed MUDAM scheme controls the interference of the second beam to the first selected user $p$ by an amount of $|\varepsilon|^{2}$ and the second user $q$ is selected in an opportunistic manner. However, since the orthogonal scheme does not have any constraint except $\mathbf{W}^{H} \mathbf{W}=\mathbf{I}$, there may not exist an user with small interference as shown in Fig. 8(b). Therefore, the orthogonal beam scheme may provide a capacity even less than the single beam scheme when the number of users is small.

Fig. 9 depicts the performance of the proposed MUDAM scheme applied to $(4 \times 1)$ MISO system. It can be seen that the capacity of $(4 \times 1)$ system with two beams is nearly same as that of $(2 \times 1))$ system with two beams. Since the capacity of the proposed MUDAM scheme increases as the number of beams increases, it is desirable to use multiple beams as many as the number of antennas for maximum improvement of the capacity.

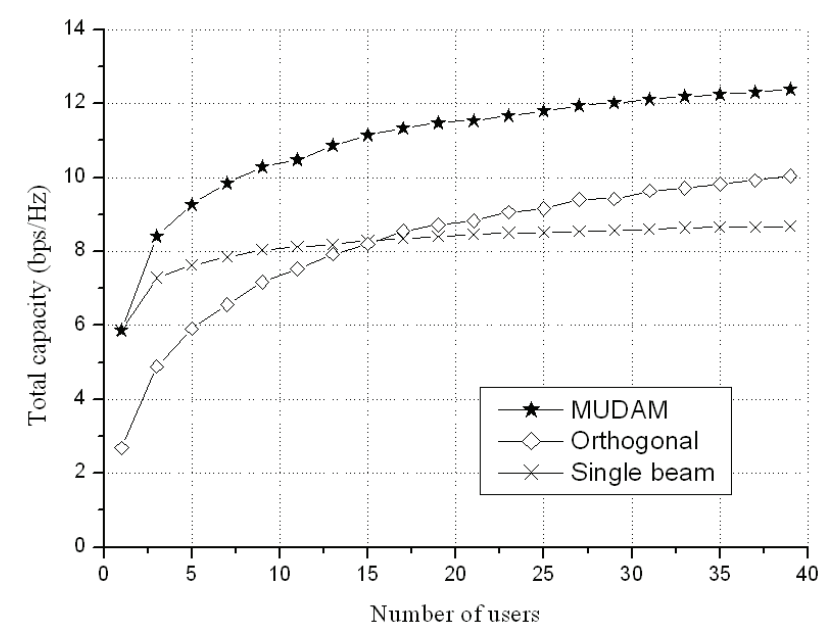

Fig. 7. The capacity of $(2 \times 1)$ MISO systems in Rayleigh fading channel at $20 \mathrm{~dB}$ SNR.

\section{Conclusions}

In this paper, we have proposed a new multiple antenna transmission scheme that can simultaneously achieve the diversity and multiplexing gain by generating multiple random beams. The multiple random beams are generated so that each beam interferes with other beams in a controlled manner. Simulation results show that the proposed MUDAM scheme can provide a system capacity larger than the single beam and orthogonal multi-beam schemes. The proposed scheme can provide a substantial capacity gain in multi-user and multiantenna systems.

\section{APPENDIX A}

\section{CAPACITY GAIN By MUDAM}

Consider the MUDAM scheme in an $(M \times 1)$ MISO system. Assume that the base station employs $B$ beams, where $1 \leq B \leq M$. In the MUDAM scheme using $i$ beams, $i=1,2, \ldots, B$, the SINR of user $k$ through the $j$-th beam can be represented as

$$
\operatorname{SINR}_{k, j, i}=\frac{\frac{1}{i}\left|\mathbf{h}_{k}^{H} \mathbf{w}_{j}\right|^{2}}{\sigma^{2}+(i-j) \frac{1}{i}|\varepsilon|^{2}+\frac{1}{i} \sum_{n=1}^{j-1}\left|\mathbf{h}_{k}^{H} \mathbf{w}_{n}\right|^{2}}
$$




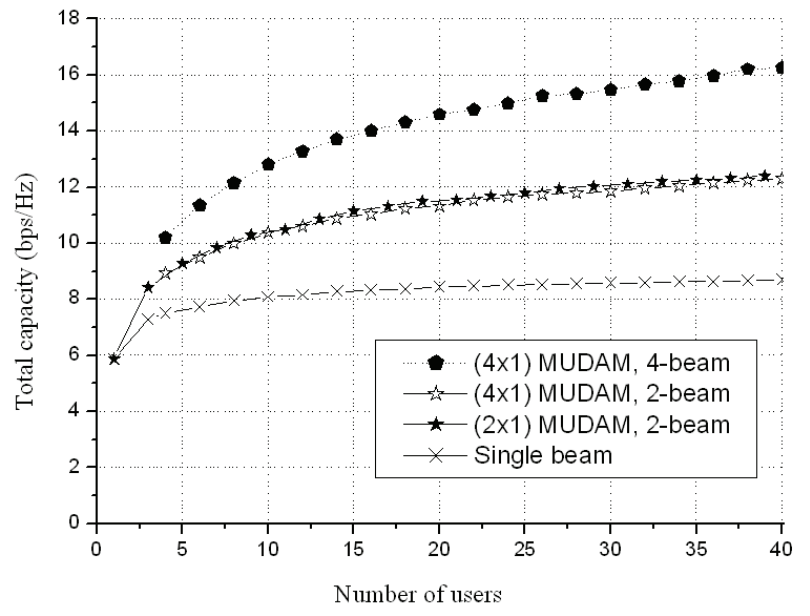

Fig. 9. The capacity of $(4 \times 1)$ MISO systems with two beams and four beams in Rayleigh fading channel at 20dB SNR.

Here, $1 \leq i \leq B$ and $1 \leq j \leq i$.

Note that the transmit power of each beam is divided by $i$. The user of the $j$-th beam has uncontrollable interference from the first through $(j-1)$-th beams and controllable interference from the $(j+1)$-th through $i$-th beam. The MUDAM scheme choose the maximum SINR for the $j$-th beam as

$$
Z_{i, j}=\max \left\{\operatorname{SINR}_{1, j, i}, \operatorname{SINR}_{2, j, i}, \ldots, \operatorname{SINR}_{K, j, i}\right\} .
$$

Let the denominator and numerator of $\operatorname{SINR}_{k, j, i}$ be $S_{D}$ and $S_{N}$, respectively. Then the random variable $S_{N}$ can be modeled as a $2^{\text {nd }}$-order Chi-square random variable same as the single beam case. The random variable $S_{D}$ can be modeled as a $2(j-1)$-th order Chi-square random variable plus a constant $\left(\sigma^{2}+(i-j)|\varepsilon|^{2} / i\right)$. Therefore, $\operatorname{SINR}_{k, j, i}$ can be modeled as the division of two random variables $S_{D}$ and $S_{N}$. Assuming that $f_{S_{D}}$ and $f_{S_{N}}$ are the $p d f$ of $S_{D}$ and $S_{N}$, respectively, the $p d f$ of $\operatorname{SINR}_{k, j, i}$ can be calculated as [18]

$$
f_{\operatorname{SINR}_{k, j, i}}(\gamma)=\int_{0}^{\infty} \frac{1}{w^{3}} f_{S_{D}}\left(\frac{1}{w}\right) f_{S_{N}}\left(\frac{\gamma}{w}\right) d w .
$$

The $p d f$ of $Z_{j, i}$ can be calculated as [19]

$$
f_{Z_{j, i}}(z)=k\left(F_{\operatorname{SINR}_{k, j, i}}(z)\right)^{K-1} f_{\operatorname{SINR}_{k, j, i}}(z)
$$

where $F_{\mathrm{SINR}_{k, j, i}}$ is the distribution function of $\operatorname{SINR}_{k, j, i}$. The capacity of the $j$-th beam $E_{Z_{j, i}}\left\{C\left(Z_{j, i}\right)\right\}$ can be calculated as

$$
E_{Z_{j, i}}\left\{C\left(Z_{j, i}\right)\right\}=\int_{0}^{\infty} \log _{2}(1+z) f_{Z_{j, i}}(z) d z .
$$

The total capacity of the MUDAM scheme using $B$ beams can be represented as

$$
\begin{aligned}
C_{B} & =E\left\{\begin{array}{c}
P_{1} C\left(Z_{1,1}\right)+ \\
P_{2}\left[C\left(Z_{1,2}\right)+C\left(Z_{2,2}\right)\right]+\ldots+P_{B} \sum_{j=1}^{B} C\left(Z_{j, i}\right)
\end{array}\right\} \\
& =\sum_{i=1}^{B} \sum_{j=1}^{i} P_{i}\left[E_{Z_{j, i}}\left\{C\left(Z_{j, i}\right)\right\}\right]
\end{aligned}
$$

where $P_{i}$ is the probability that the capacity of the MUDAM scheme using $i$ beams is larger than that using $j(\neq i)$ beams. As an example, we analyze the capacity of the MUDAM scheme using two beams in Rayleigh fading channel.

When the base station uses a single beam (i.e., $B=1$ ), the instant capacity is given by

$$
C\left(Z_{1,1}\right)=\log _{2}\left(1+Z_{1,1}\right)
$$

where

$$
Z_{1,1}=\max _{k=1, \ldots, K}\left\{\frac{\left|\mathbf{h}_{k}^{H} \mathbf{w}_{1}\right|^{2}}{\sigma^{2}}\right\} .
$$

When the base station uses two beams (i.e., $B=2$ ), the instant capacity of the first beam is given by

$$
C\left(Z_{1,2}\right)=\log _{2}\left(1+Z_{1,2}\right)
$$

where

$$
Z_{1,2}=\max _{k=1, \ldots, K}\left\{\frac{\left|\mathbf{h}_{k}^{H} \mathbf{w}_{1}\right|^{2} / 2}{\sigma^{2}+|\varepsilon|^{2} / 2}\right\}
$$

and the instant capacity of the second beam is given by

$$
C\left(Z_{2,2}\right)=\log _{2}\left(1+Z_{2,2}\right)
$$

where

$$
Z_{2,2}=\max _{k=1, \ldots, K}\left\{\frac{\left|\mathbf{h}_{k}^{H} \mathbf{w}_{1}\right|^{2} / 2}{\sigma^{2}+\left|\mathbf{h}_{k}^{H} \mathbf{w}_{1}\right|^{2} / 2}\right\} .
$$

The average capacity $E_{Z_{1,1}}\left\{C\left(Z_{1,1}\right)\right\}$ and $E_{Z_{1,2}}\left\{C\left(Z_{1,2}\right)\right\}$ can be calculated using

$$
\begin{aligned}
C & =E_{Z}\left\{\log _{2}\left(1+\frac{z}{\sigma^{2}}\right)\right\} \\
& =\frac{K}{\log ^{2}} \sum_{j=0}^{K-1} C(K-1, j)(-1)^{j+1} \\
& \frac{e^{(j+1) \sigma^{2}}}{j+1} \operatorname{Ei}\left[-(j+1) \sigma^{2}\right]
\end{aligned}
$$

where $\operatorname{Ei}[x]=-\int_{-x}^{\infty}\left(e^{-t} / t\right) d t$, since $Z_{1,1}$ and $Z_{1,2}$ can be modeled as $2^{\text {nd }}$-order Chi-square random variables. The average capacity of the second beam $E_{Z_{2,2}}\left\{C\left(Z_{2,2}\right)\right\}$ can be calculated by

$$
E_{Z_{2,2}}\left\{C\left(Z_{2,2}\right)\right\}=\int_{0}^{\infty} \log _{2}(1+z) f_{Z_{2,2}}(z) d z
$$

where, from (A. 3) and (A. 4), the $p d f$ of $Z_{2,2}$ is represented as

$$
f_{Z_{2,2}}(z)=K e^{-\sigma^{2} z} \frac{\left(1+z-e^{-\sigma^{2} z}\right)^{K-1}\left(1+\sigma^{2}+\sigma^{2} z\right)}{(1+z)^{K+1}} .
$$

As mentioned before, the base station needs to use a single beam if

$$
C\left(Z_{1,1}\right)>C\left(Z_{1,2}\right)+C\left(Z_{2,2}\right) .
$$

The probability $P_{2}$ is defined as

$$
P_{2}=\operatorname{Prob}\left\{C\left(Z_{1,1}\right)<C\left(Z_{1,2}\right)+C\left(Z_{2,2}\right)\right\}=1-P_{1} .
$$


It can be shown that

$$
\begin{aligned}
P_{2}=\operatorname{Prob}\left\{\begin{array}{c}
\log _{2}\left(1+\frac{\left|\mathbf{g}_{1}^{H} \mathbf{w}_{1}\right|^{2}}{\sigma^{2}}\right) \\
<\log _{2}\left(1+\frac{\left|\mathbf{g}_{1}^{H} \mathbf{w}_{1}\right|^{2} / 2}{\sigma^{2}+|\varepsilon|^{2}}\right) \\
+\log _{2}\left(1+Z_{2,2}\right)
\end{array}\right\} \\
=\operatorname{Prob}\left\{Z_{2,2}>\eta\right\} \\
=\int_{\eta}^{\infty} f_{Z_{2,2}}(z) d z
\end{aligned}
$$

where

$$
\eta=\frac{\left(\sigma^{2}+|\varepsilon|^{2}\right)\left(2 \sigma^{2}+\left|\mathbf{g}_{1}^{H} \mathbf{w}_{1}\right|^{2}\right)}{\sigma^{2}\left\{2\left(\sigma^{2}+|\varepsilon|^{2}\right)+\left|\mathbf{g}_{1}^{H} \mathbf{w}_{1}\right|^{2}\right\}}-1 .
$$

To have some intuition, assume that $|\varepsilon|^{2}, \sigma^{2} \ll 1$. The we have

$$
P_{2} \approx 1-2^{-K}
$$

which implies that the use of multiple beams results in performance improvement over the use of a single beam as the number of users increases. Note that (A.14) and (A.18) cannot be represented in a closed form.

The capacity of MUDAM scheme with two beams is given by

$$
\begin{aligned}
C_{2} & =\sum_{i=1}^{2} \sum_{j=1}^{i} P E_{Z_{j, i}}\left\{C\left(Z_{j, i}\right)\right\} \\
& =P_{1} E_{Z_{1,1}}\left\{C\left(Z_{1,1}\right)\right\} \\
& +P_{2}\left[E_{Z_{1,2}}\left\{C\left(Z_{1,2}\right)\right\}+E_{Z_{2,2}}\left\{C\left(Z_{2,2}\right)\right\}\right]
\end{aligned}
$$

Fig. 6 depicts the capacity of $(2 \times 1)$ MISO systems in Rayleigh fading channel at $20 \mathrm{~dB}$ SNR where $\varepsilon=0.01$ for the MUDAM scheme. It can be seen that the use of the proposed MUDAM scheme outperforms the single beam scheme. Note that the discrepancy between the analysis and simulation results in the MUDAM scheme is mainly due to the approximation of SINR $_{k, 2,2}$ as a division of two independent $2^{\text {nd }}$-order Chisquare random variables.

\section{REFERENCES}

[1] G. J. Foschini and M. J. Gans, "On limits of wireless communications in a fading environment when using multiple antennas," Wireless Pers. Commun., vol. 6, no. 3, pp. 311-335, June 1998.

[2] G. D. Golden, G. J. Foschini, V. A. Valenzuela, and P. W. Wolniansky, "Detection algorithm and initial laboratory results using V-BLAST space-time communication architecture," IEE Electron. Lett., vol. 35, pp. 14-16, Jan. 1999.

[3] S. M. Alamouti, "A simple transmit diversity technique for wireless communications," IEEE J. Select. Areas Commun., vol. 16, no. 8, pp. 1451-1458, Oct. 1998.

[4] G. Lebrun, T. Ying, and M. Faulkner, "MIMO transmission over a timevarying TDD channel using SVD," IEE Electron. Lett., vol. 37, no. 22, pp. 1363-1364, Oct. 2001.

[5] Z. Lizhong and D. N. C. Tse, "Diversity and multiplexing: A fundamental tradeoff in multiple-antenna channels," IEEE Trans. Inform. Theory, vol. 49, no. 5, pp. 1073-1096, May 2003.
[6] R. W. Heath Jr., M. Airy, and A. J. Paulraj, "Multiuser diversity for MIMO wireless systems with linear receivers," in Proc. Asilomar Conf. Signals, Systems and Computers, Nov. 2001, vol. 2, pp. 1194-1199.

[7] W. Rhee, W. Yu, and J. M. Cioffi, "Utilizing multiuser diversity for multiple antenna systems," in Proc. IEEE Wireless Commun. Network. Conf., Sep. 2000, vol. 1, pp. 420-425.

[8] D. J. Mazzarese and W. A. Krzymien, "High throughput downlink cellular packet data access with multiple antennas and multiuser diversity," in Proc. Veh. Technol. Conf.-Spring, Apr. 2003, vol. 2, pp. 1079-1083.

[9] R. Gozali, R. M. Buehrer, and B. D. Woerner, "The impact of multiuser diversity on space-time block coding," IEEE Commun. Lett., vol. 7, no. 5, pp. 213-215, May 2003.

[10] R. Gozali, R. M. Buehrer, and B. D. Woerner, "On the performance of scheduling over space-time architectures," in Proc. IEEE Veh. Technol. Conf.-Fall, Sep. 2002, vol. 1, pp. 415-419.

[11] P. Viswanath, D. N. C. Tse, and R. Laroia, "Opportunistic beamforming using dumb antennas," IEEE Trans. Inform. Theory, vol. 48, no. 6, pp. 1277-1294, June 2002.

[12] J. Chung, C. Hwang, K. Kim, and Y. K. Kim, "A random beamforming technique in MIMO systems exploiting multiuser diversity," IEEE $J$. Select. Areas Commun., vol. 21, no. 5, pp. 848-855, June 2003.

[13] V. K. N. Lau, "Proportional fair spatial scheduling for wireless access point with multiple antenna-Reverse link with scalar feedback," in Proc. IEEE Global Commun. Conf., Nov. 2002, vol. 1, pp. 763-767.

[14] D. Wu and R. Negi, "Utilizing multiuser diversity for efficient support of quality of service over a fading channel," in Proc. IEEE Int. Conf. Commun., May 2003, vol. 3, pp. 2202-2207.

[15] M. Costa, "Writing on dirty paper," IEEE Trans. Inform. Theory, vol. 29, no. 3, pp. 439-441, May 1983.

[16] G. Caire and S. Shamai, "On the achievable throughput of a multiantenna Gaussian broadcast channel," IEEE Trans. Inform. Theory, vol. 49, no. 7, pp. 1691-1706, July 2003.

[17] M. Shubert and H. Boche, "Joint "dirty paper" pre-coding and downlink beamforming," IEEE Int. Symp. Spread-Spectrum Technol., Sep. 2002, pp. 536-540.

[18] P. Z. Peebles, Jr., Probability, Random Variables, and Random Signal Principles. New York: McGraw-Hill, 1993.

[19] [Online] Available: http://www.math.uah.edu/statold/sample/sample7.html

[20] M. Sharif and B, Hassibi, "On the capacity of MIMO broadcast channel with partial side information," in Proc. 37th Asilomar Conference on Signals, Systems and Computers, Nov. 2003, vol. 1, pp. 958-962.

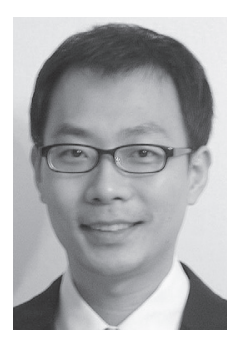

Sung-Soo Hwang received the B.S., M.S. and Ph.D. degrees from Seoul National University, Seoul, Korea in 1996, 1998 and 2004, respectively, in electrical engineering and computer science. He is currently a senior engineer in the Telecommunication R\&D Center, Samsung Electronics, and his current interest is in the area of wideband wireless communication systems including MIMO detection, multiuser diversity and multi-user MIMO, etc.

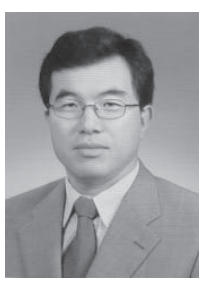

Yong-Hwan Lee received the B.S. degree from Seoul National University, Seoul, Korea in 1977 , M.S. degree from Korea Advanced Institute of Science \& Technology in 1980 and Ph.D. degree from the University of Massachusetts, Amherst, in 1989. From 1980 to 1985, Yong-Hwan Lee was a Senior Researcher at the Korea Agency for Defense Development (ADD), Daejon. From 1987 to 1989, he was a Software Consultant in the Engineering Computer Services, Univ. of Massachusetts, Amherst. From 1989 to 1994 , he was a Principal Engineer at Motorola, Inc. (Mansfield, MA). In 1994, he joined the School of Electrical Engineering, Seoul National University, as a faculty member. His current interest is in the areas of wired/wireless signal transmission systems, robust signal processing, and detection and estimation. 